\title{
Perception of pain: an investigation of caffeine's impact on inflammatory conditions
}

\section{Editorial}

Advanced science students in Australia have carried out experimental research on health and well-being this past year at the Circular Head Christian School located in the state of Tasmania. In one of these experiments, spanning all of 2014 academic year (February through December) the effect of caffeine on pain was investigated. The research entitled "Perception of Pain as a Function of Caffeine intake: An exploratory study" was carried out by Ms. Shona Poke for her Advanced Science Project. As a vasodilator, caffeine is currently being used to enhance delivery efficacy of medications such as ibuprofen and paracetamol. However, several cases have been reported with higher doses of caffeine potentially increasing pain in medical conditions involving inflammation, such as tendonitis, bursitis, endometriosis and arthritis. Ms. Poke felt these cases warranted further scientific investigation.

In order to test the hypothesis that caffeine increases perception of pain with higher doses, volunteer subjects were recruited for the experiment, whohad a history of inflammation and previous symptoms of pain in the afflicted area. A Within Subjects experimental design was used, where all Subjects were included in all conditions of the experiment. Subjects were also matched for age, tolerance to pain, and medical history. The two independent variables were 1) level of caffeine consumed daily, and 2) level of exercise/activity per day. There were three levels of the first Independent Variable (IV-1) with three levels of caffeine being consumed: $0 \mathrm{mg}, 30 \mathrm{mg}$, and $60 \mathrm{mg}$. Each level was consumed for two weeks. The second Independent Variable (IV-2) had two levels, with: 1) little or no activity, or 2) high level of activity. These levels were operationally defined for the subjects in terms of both intensity and duration of a variety of activities within each level. Each level was maintained over a three week period, spanning all three levels of caffeine intake. The Dependent Variable was Level of Perceived Pain on a 10-point scale based upon the Importance of Pain Scale as published in Remote Medicine Ireland (17 Oct 2011).

Results show that a clear interaction effect between level of caffeine consumed and level of activity produced a wide variation in perceived pain, with higher levels of activity and higher levels of caffeine consumed resulting in the highest perceived levels of pain, and lowest levels of caffeine and low levels of activity resulting in the lowest reports of perceived pain.
Volume 2 Issue I - 2015

\author{
Nadira T Pardo \\ Psychology Department, California Southern University, \\ Australia
}

Correspondence: Nadira T Pardo, Psychology Department, California Southern University, USA,

Email nadira.pardo@my.calsouthern.edu

Received: January 30, 2015 | Published: February 17, 2015

Thus, although caffeine is successfully being used to facilitate timely delivery of medication in the body for such medications as mild over-the-counter pain relief, the results from this study suggest that the vasodilator mechanisms which allow such expedient delivery throughout the body may also contribute to increased inflammation in pre-existing medical conditions with inflammation. For example, if someone is suffering from mild pain from tennis elbow (tendonitis) and takes an over-the-counter medication such as ibuprofen or paracetamol which has a large amount of caffeine in it to expedite the delivery of the pain relief medication, the end result may be that the perceived pain actually increases rather than reduces as the inflammation will become worse due to the normal properties of the caffeine. This research was exploratory in nature, and is based on case studies, so further research is required with larger sample sizes to increase overall validity and reliability of these initial findings. ${ }^{1}$

\section{Acknowledgements}

None.

\section{Conflict of interest}

The author declares no conflict of interest.

\section{References}

1. The importance of the Pain Scale. Remote Medicine Ireland; 2011. 